brightness distribution observed at $6-\mathrm{cm}$ with the VLA can be described by two distinct components: a) an extended diffuse structure, which closely reproduces the shape of the observable nebulosity, and b) a sharp bright arc feature on the east side of the extended component, unresolved towards the outside at the resolution of $\sim 4 "$.

From CO maps of the same region we know that on the east side the nebula is bounded by a high density molecular cloud (see also Wramdemark, Lynga, and Johansson, paper presented at this symposium). To reproduce the surface brightness observed in the radio continuum, we have used a three dimensional model in which the ionized gas distribution is determined by gas outflow from a spherical molecular blob. The ionization is produced by an early type star placed outside the blob. The whole complex is also surrounded by a low density diffuse region.

To derive the electron density distribution we made the following assumptions: a) at the ionization front (assumed to be spherical) the $\mathrm{n}_{\mathrm{e}}$ is determined by the stellar ionizing flux reaching the surface, which, in turn, is determined by geometrical factors and by the gas absorption along the path, and b) the ionized gas is assumed to expand radially at constant velocity and to be at constant temperature.

In addition to the ne produced by the erosion of the molecular cloud, a "standard" Strömgren region due to the diffuse surrounding component is also added.

This simple model can fit the observed surface brightness distribution quite well. In particular, the required UV photon flux is $8.4 \times$ $10^{44} \mathrm{~s}^{-1}$ (equivalent to an 09 ZAMS) of which only a fraction is absorbed in the outflow. The remaining UV photons are absorbed in the diffuse component. The distance of the exciting star from the molecular blob (assumed to have a radius of $0.3 \mathrm{pc}$ ) is $0.34 \mathrm{pc}$. It is remarkable that the position of the exciting star derived in this way closely matches that of a 2 micron source (Manpaso, private communication) which is the proposed candidate for the ionizing star of the nebula.

The maximum electron density of the ionization front turns out to be $1.1 \times 10^{3} \mathrm{~cm}^{-3}$ and the erosion of the molecular blob due to the ionization is $4 \times 10^{-5} \mathrm{M}_{\odot} \mathrm{yr}^{-1}$. If this rate has been constant since the turn on of the early type star, the total mass of the diffuse HII region can be produced by the erosion over a time scale of $5 \times 10^{5}$ years.

\title{
EXPANSION OF ULTRA-COMPACT H II REGIONS
}

J.M. Moran, M.J. Reid, B. Sams

Harvard-Smithsonian Center for Astrophysics, USA

The dynamical properties of the ionized envelopes surrounding massive, newly formed stars can be probed by the measurement of radio recombination lines over a wide range of frequencies. In an expanding 
envelope, the centroids of the radio recombination lines move progressively to lower velocities with increasing value of the principal quantum number, $n$. This shift is caused by the free-free absorption on the near side of the envelope combined with non-LTE radiative transfer effects.

We have observed the $\mathrm{H} 66 \alpha, \mathrm{H} 76 \alpha$, and $\mathrm{H} 110 \alpha$ lines towards $\mathrm{W} 3(\mathrm{OH})$ with the VLA. The measured line velocities of $-49.1 \pm 0.3,-50.5 \pm 0.3$, and $-68.3 \pm 4 \mathrm{~km} \mathrm{~s}^{-1}$, respectively, and other line parameters were analyzed with a non-LTE radiative transfer model. Preliminary results suggest an expansion velocity of about $20 \mathrm{~km} \mathrm{~s}^{-1}$ and a stellar velocity of about $-46 \mathrm{~km} \mathrm{~s}^{-1}$. The $\mathrm{H} 66 \alpha$ and $\mathrm{H} 76 \alpha$ lines show significant departures from Gaussian profiles due to pressure broadening, and the $H 110 \alpha$ line has a nearly Lorentzian profile, requiring a mean density of about $2 \times 10^{5} \mathrm{~cm}^{-3}$ independent of the excitation temperature. We also observed the recombination lines towards W49S and G10.6-0.4 in the H66 and $\mathrm{H} 76 \alpha$ lines but detected no shift in velocity between the lines in either source, presumably because of smaller expansion velocities.

HOLLOW H II REGIONS: FROM GIANT TO ULTRACOMPACT

T. Montmerle, H. Dorland

Centre d'Etudes Nucléaires de Saclay, France

C. Doom

Astrofysisch Instituut, Vrije Universiteit Brussel, Belgium

\section{PROBLEM AND RECENT DEVELOPMENTS}

$H$ II regions around $O B$ associations have a thick-shell structure (see, e.g., the Carina and Rosette nebulae), and yet the standard "Hot Interstellar Bubble" model (e.g., Weaver et al. 1977) predicts thin H II she11s around a large X-ray emitting volume, when associated with stellar winds. Observations suggest that strong dissipation must occur at the edge of the wind cavity: (i) expansion velocities there are much smaller than predicted by the standard model (e.g., Chu, 1983); (ii) in bubbles around WR stars, overabundances of $\mathrm{N}$, He, etc., are seen, hence the need to cool these WR-produced elements down to observable temperatures (Kwitter, 1981). Also, two theoretical developments are important: (i) new stellar evolution models for massive stars, including mass loss and overshooting in convective cores (e.g., Doom, 1985); (ii) a non-linear theory for heat conduction with steep temperature gradients (Luciani et al. 1985). 